\title{
POSITRON DEPTH PROFILING IN SOLID SURFACE LAYERS
}

\author{
Robert I. GRYNSZPAN ${ }^{\mathrm{a}}$, Wolfgang ANWAND ${ }^{\mathrm{b}}$, Gerhard BRAUER ${ }^{\mathrm{b}}$, Paul G. COLEMAN ${ }^{\mathrm{c}}$
}

a) Dept. Lasers, Optics and Thermo-optics, DGA, 94114 Arcueil, and CNRS-ISCSA-Thiais, France.

b) Forschungszentrum Dresden-Rossendorf, Postfach 510119, 013114 Dresden, Germany.

c) Dept. of Physics, University of Bath, Bath BA2 7AY, United Kingdom.

\begin{abstract}
We briefly review the principles of the Doppler Broadening of the positron annihilation radiation line, the most common technique used in defect depth profiling of solids relevant to dcbeams. We focus on some specific examples of Slow Positron Implantation Spectroscopy (SPIS) investigations related to technological issues such as, for instance, i) phase transitions in metal coatings possibly induced by internal stresses, ii) substrate pre-treatment or annealing dependence of defect profiles at metal/polymer interfaces or in the deposited layers, and. iii) near-surface structural modification by ion implantation in ceramics and multilayers. In each case we elaborate on the possibility of using SPIS results and possible depth profile features as criteria for on- or off-line quality control in industrial processes. We finally conclude with an overall picture of the operating characteristics of positron annihilation techniques.
\end{abstract}

Résumé - Profils d'implantation des positons dans des couches de surface des solides. Nous passons brièvement en revue les principes de la méthode de l'élargissement Doppler de la raie d'annihilation des positons, technique la plus fréquemment utilisée en analyse de surface des solides par faisceau à courant continu. Nous considérons quelques d'applications technologiques de la caractérisation par spectroscopie d'implantation des positrons lents (SPIS), telles que, par exemple, i) les transitions de phases éventuellement induites par contraintes internes dans les dépôts métalliques, ii) l'influence de prétraitements du substrat ou de recuits sur le profil des défauts aux interfaces métal/polymère ou dans des couches déposées, et iii) la modification structurale par implantation ionique en proche surface de céramiques et de multicouches. Dans tous les cas nous envisageons l'éventualité d'utiliser la technique SPIS et les particularités des profils de défauts comme critères dans des contrôles de qualité en ligne ou en post-production industrielle. Enfin, nous concluons par un panorama des caractéristiques de mise en œuvre des techniques de spectroscopie d'annihilation des positrons.

Reprints: Dr. G. Brauer, FZD-Rossendorf, Postfach 510119, 01314 Dresden, Germany, g.brauer@fzd.de 


\section{$\underline{\text { INTRODUCTION }}$}

Important properties of solids ultimately depend on both their electronic and defect structures. Therefore, an experimental and maybe even theoretical estimation of these structures is a major task of materials science. The positron is a relevant atomic probe, as its resulting gamma photons carry information about the localization sites of the probe and thus about the electrons of the solid under investigation. Furthermore, positrons may get trapped in open-volume defects until they annihilate and exhibit a specific sensitivity to these types of defects. Thus, in principle all methods of positron annihilation spectroscopy (PAS), i.e. Lifetime (LT), Angular Correlation of the Annihilation Radiation (ACAR), and Doppler Broadening (DB), are suited to reveal electronic and structural properties of a solid [1-3].

Positrons are the antiparticles of electrons, and when they encounter each other both particles annihilate into gamma rays. The annihilation process itself is fully described by quantum electrodynamics, and includes the application of the conservation laws of physics. Among the latter, the Einstein formula $\mathrm{E}=\mathrm{mc}^{2}$ is the best known and plays a major role. If the annihilation process happens in solids, most probably two annihilation photons of $511 \mathrm{keV}$ are emitted simultaneously into opposite directions. Due to conservation of the total momentum of the annihilating electronpositron pair, which is determined only by the electron momentum to first order, i) a very small but measurable deviation from collinearity of the annihilation quanta (angular correlation), and ii) broadening of the annihilation line (Doppler broadening) occur. In this review, we focused on this latter effect to study various properties of solid surface layers.

\section{TECHNICALITIES}

\subsection{Choice of positrons}

The use of positrons as probes of solids depends on their origin. When emerging from natural beta decay of the isotope ${ }^{22} \mathrm{Na}$ (half-life $\sim 2.6 \mathrm{y}$ ), they have a kinetic energy ranging from 0 to $542 \mathrm{keV}$. As a consequence, if directly used, they are stopped with a given depth distribution depending on the density $\rho$ of the solid under investigation. Their maximum penetration depth $z_{\max }$ can be calculated to a good approximation as $z_{\max }=10^{3} / \rho(\mu \mathrm{m})$, if $\rho$ is given in $\mathrm{g} / \mathrm{cm}^{3}$. In the case of mono-energetic positrons, much narrower depth distributions can be achieved. For comparison,

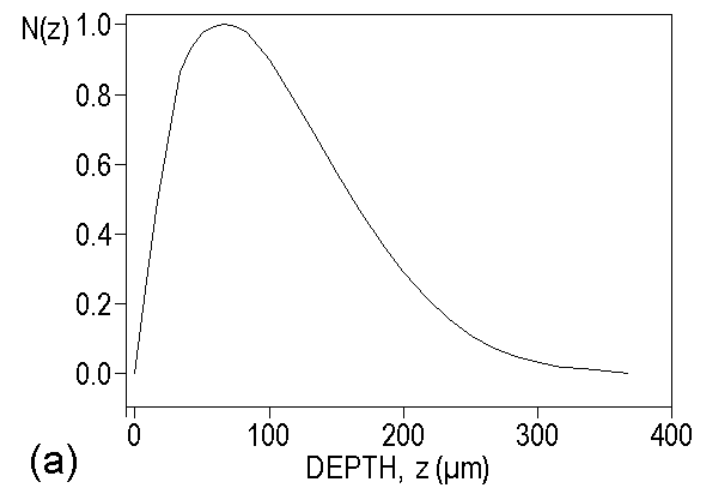

Figure 1. Depth distribution of thermalized positron in $\mathrm{SiC}$ for (a) "natural" positrons from ${ }^{22} \mathrm{Na}$, and (b) mono-energetic positrons of energy $E$ as indicated. $P(E, z)$ is the distribution function of positrons having the energy $E$, whereas $N(z)$ is the distribution function obtained by integration of $P(E, z)$ over all energies $(0-542 \mathrm{keV})$ of positrons from ${ }^{22} \mathrm{Na}$. 
both cases are exemplified in figure 1 for silicon carbide having a density $\rho=3.2 \mathrm{~g} / \mathrm{cm}^{3}$.

Simulated mono-energetic positron implantation profiles $[4,5]$ can be fairly well parameterized by a so-called Makhov distribution $P(E, z)$ as follows:

$$
P(E, z)=\left(\mathrm{m} / z_{\mathrm{o}}\right)\left(z / z_{\mathrm{o}}\right)^{\mathrm{m}-1} \exp \left[-\left(z / z_{\mathrm{o}}\right)^{\mathrm{m}}\right]
$$

where $z_{o}=1.13<z>$, and $<_{z}>=\alpha_{\mathrm{p}} / \rho E^{\mathrm{n}}=$ mean depth, with $E$ being the positron energy in $\mathrm{keV}$, $\rho$ the density of the solid in $\mathrm{g} / \mathrm{cm}^{3}$, and $\alpha_{\mathrm{p}}, \mathrm{m}$, and $\mathrm{n}$ material independent parameters. The latter parameters were reported [6] to have the following accurate values: $\mathrm{m}=2(0.1), \mathrm{n}=1.62(0.05)$, and $\alpha_{\mathrm{p}}=4.0(0.3) \mu \mathrm{g} \mathrm{cm} \mathrm{cm}^{-2} \mathrm{keV}^{-1.62}$. From further intensive research it was concluded that a value $\alpha_{p}=3.6$ should be more accurate [7], and therefore this more realistic value is generally applied throughout this work.

If bulk properties of a solid are to be revealed, the direct use of natural positrons from ${ }^{22} \mathrm{Na}$ is completely sufficient because the information carried by the annihilation photons does not depend on the depth where the annihilation takes place. However, materials of choice are often modified in a shallow layer near their surface, or are provided as a thin film covering a substrate. Therefore, Slow Positron Implantation Spectroscopy (SPIS), based on the generation, implantation and subsequent annihilation of mono-energetic positrons in a sample, has to be used to study depthdependent properties of the material, and in particular the depth profile of vacancy-type damage. Comprehensive summaries of earlier [8] and more recent developments [9] in this field have already been published.

\subsection{Positron beam layout}

For a beam source based on the beta decay of an isotope (e.g. $\left.{ }^{22} \mathrm{Na}\right)$, the generation of monoenergetic positrons starts with natural positrons, which are hitting a so-called positron moderator. This moderator, a solid with a "strong" negative work function $\left(\phi_{+}\right)$for positrons, commonly consists of a well-annealed, mono-crystalline tungsten foil of about $2 \mu \mathrm{m}$ thickness $\left(\phi_{+\mathrm{W}} \approx-3 \mathrm{eV}\right)$. Due to this unusual property, a positron stopped in this tungsten foil and diffusing to its surface will be spilled out with a kinetic energy of $\sim 3 \mathrm{eV}$. It has to be mentioned that the efficiency of this process is only of the order of $10^{-4}$. About $13 \%$ of all positrons emitted by the ${ }^{22} \mathrm{Na}$ source are stopped and annihilated in the tungsten foil, whereas about $87 \%$ are transmitted through the foil because their kinetic energy is too high to be stopped and thermalized.
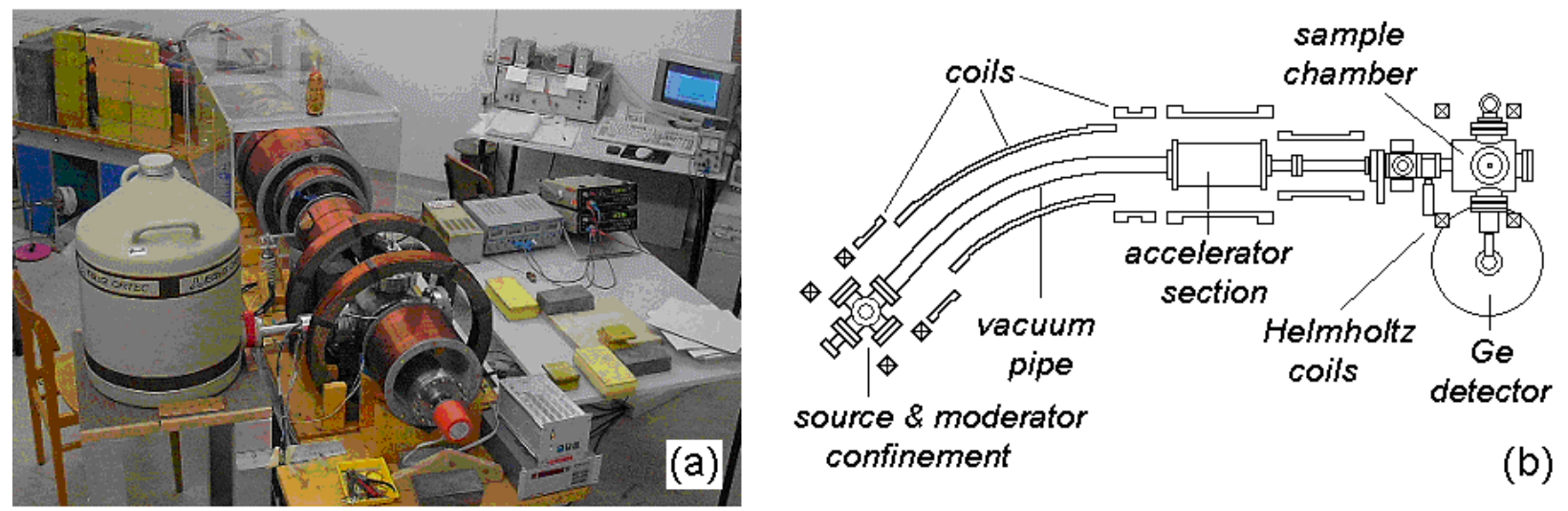

Figure 2. Continuous mono-energetic positron beamline at Forschungszentrum DresdenRossendorf: (a) overview; (b) sketch of the layout. 
To form a mono-energetic positron beam, the work function positrons have to be separated from higher energy positrons and from the gamma rays resulting from annihilations in either the moderator or the ${ }^{22} \mathrm{Na}$ source itself. In practice, in the case of the FZD-R beam (figure 2), this is done by application of a small voltage $(+30 \mathrm{~V})$ to the moderator foil in order to suck the work function positrons away, thus giving them a kick that guides them towards the sample in a vacuum of at least $10^{-6}$ Torr. A magnetic guiding field of $\sim 100 \mathrm{G}$ is produced from a set of Helmholtz and other coils wound from copper wire, and thus these positrons of very low kinetic energy easily follow a bent tube towards the sample chamber. Thereby they pass an accelerator section, where their kinetic energy may be increased in steps as low as $50 \mathrm{eV}$, if required, up to a maximum energy of $36 \mathrm{keV}$. In the given construction (figure 2), the acceleration voltage of positive sign is used to float the positron source in order to keep the sample under investigation grounded. As a result, all types of solids, including insulators, can be easily studied.

\subsection{Other possible positron moderators}

The search for efficient positron moderators has been going on for decades. Indeed, moderators having substantially higher efficiencies than the present value of $10^{-4}$ would allow one not only to shorten the required measuring time but also to make use of much weaker radioactive ${ }^{22} \mathrm{Na}$ positron sources. Solid noble gas layers approaching a conversion factor of $10^{-3}$ have been suggested [10] and are used in some beams [8,9] with, however, the burden of operating a cryogenic system near radioactive material.

The idea of using electric fields in semiconductors or insulators to increase the mobility of positrons to the emitting surface, so-called "field-enhanced" moderators, was originally suggested in 1979 [11] and discussed later in the context of modern epitaxial/semiconductor systems [12, 13]. Later, the suitability of the semiconductor properties of $6 \mathrm{H}-\mathrm{SiC}$ have been investigated [14]. Up to date, no experimental results of a working device have been reported to our knowledge.

Furthermore, the re-emission properties and time stability of tungsten alloys has been monitored. In the case of $\mathrm{Ni}_{80} \mathrm{~W}_{20}$ (amorphous in the as-prepared state), the dependence of the positron re-emission yield on annealing temperature $\left(\mathrm{T}_{\mathrm{a}}\right)$ exhibits a drastic change in surface behaviour, increasingly similar to that of tungsten with rising $\mathrm{T}_{\mathrm{a}}$. This seems to indicate a "reconstruction" of the near-surface layer in favour of $\mathrm{W}$ atoms burying Ni atoms underneath (figure 3) [15]. As this alloy
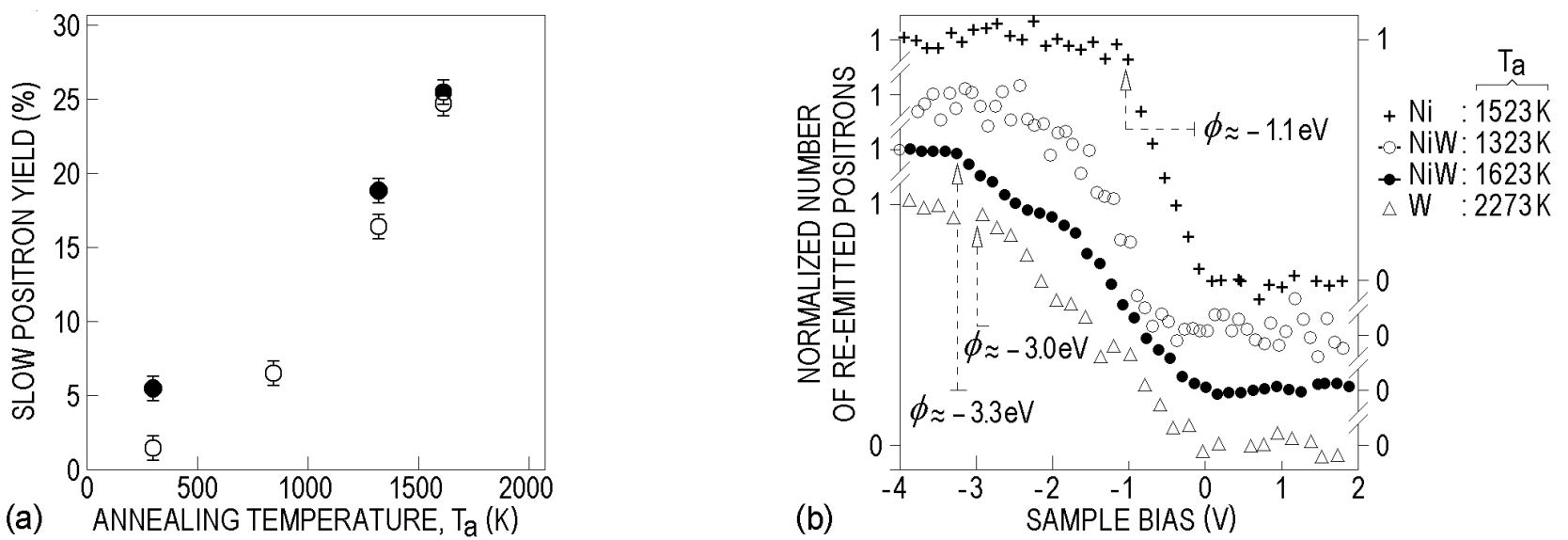

Figure 3. a) Annealing dependence of the slow positron re-emission yield from the surface of $\mathrm{Ni}_{80} \mathrm{~W}_{60}$, for $1 \mathrm{keV}$ incident positrons (measurements at room temperature, $\mathrm{T}_{\text {room }}$ ). Open symbols: asprepared sample or annealed at $\mathrm{T}_{\mathrm{a}}$; closed symbols: same sample re-annealed after storage in the air at $\mathrm{T}_{\text {room }}$ during 6 months. b) Positron re-emission vs. sample voltage bias for pure $\mathrm{Ni}$ and $\mathrm{W}$ and for $\mathrm{NiW}$ alloy, after annealing at $\mathrm{T}_{\mathrm{a}}(\phi$ : positron work function). 
is prepared by electro-deposition, it could be used to coat any desired shape designed either to fit into sections with uneasy access, e.g. in beams with on-line source production, or to improve optical collection of positrons.

Although no successful application of the above mentioned materials has been reported in the literature so far, it is worth noting that these studies have been actually performed in part at the University of East Anglia (UEA) Norwich, using a setup based on the principle of a positron reemission microscope [16]. However, such a new "positron microscopy", as well as the positroninduced Auger spectroscopy [17], are restricted to the study of ultra thin layered materials and will not lead to further development in this paper.

\subsection{Data analysis and interpretation}

The motion of the electron-positron pair prior to annihilation, which arises essentially from the electron momentum, causes the Doppler broadening of the $511 \mathrm{keV}$ annihilation line and can be characterized by the line-shape parameters $S$ and $W$. In brief, the values of $S$ and $W$ are defined by the ratio of counts in the central region and in the high-momentum region symmetrical to the annihilation gamma peak, respectively, and the total number of counts in the peak (figure 4 ). $S$ is a measure of the low momentum electron density, i.e. preferentially representing valence electrons, at the annihilation site. Meanwhile, $W\left(=W_{\mathrm{L}}+W_{\mathrm{H}}\right.$, see figure 4$)$ is a measure of the electron density of high momentum at the annihilation site, i.e. preferentially representing stronger bound core electrons. It is common, for a reference sample, to set an energy window such as to obtain $S_{\text {ref }} \sim 0.5$. In all given results, when specific indication is lacking, $S$ is normalized to the bulk value of the reference material considered. Correspondingly, a bulk value $W_{\text {ref }}$ may be defined also, depending on the material considered.

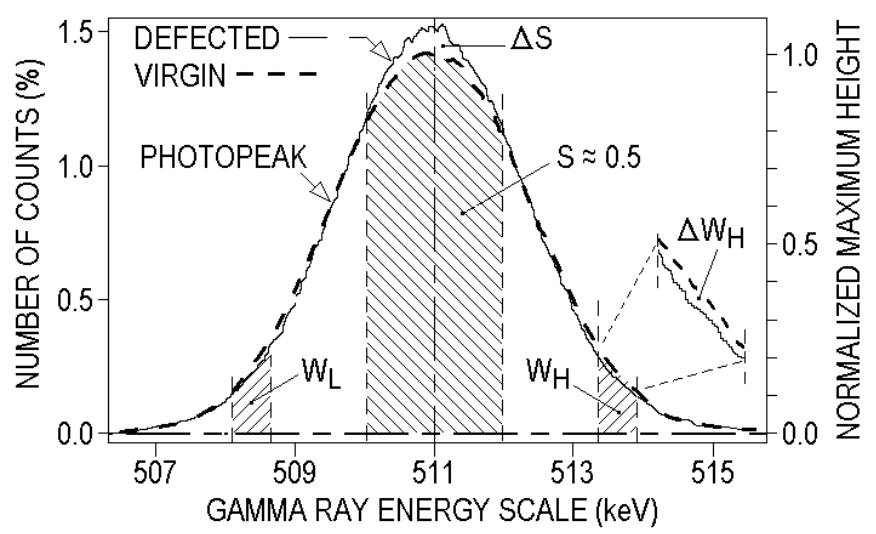

Figure 4. Example of energy window settings limiting the $S$ and $\mathrm{W}$ areas for a defected sample ( $\alpha$-irradiated zirconia) compared to a reference sample ("virgin": unirradiated zirconia). The number of counts is normalized to the total area of the photopeak, after subtraction of the background due to Compton scattering.

The depth distribution of thermalized positrons initially implanted at a given energy $E$, which can be described by a Makhovian profile (see figure 1), may become distorted due to positron diffusion and trapping prior to annihilation. It is understood that each point of a measured $S(E)$ dependence represents an integral over this final positron distribution at any given positron energy $E$. The derivation of physical information from $S(E)$ is generally performed by fitting $S(E)$, e.g., assuming that the sample may consist of a certain number of homogeneous layers, each of them characterized by a given depth width, mean $S$ parameter, and positron diffusion length $L_{+}[18]$. The absolute value of $L_{+}$in a layer is strongly dependent on the presence of impurities and defects in the 

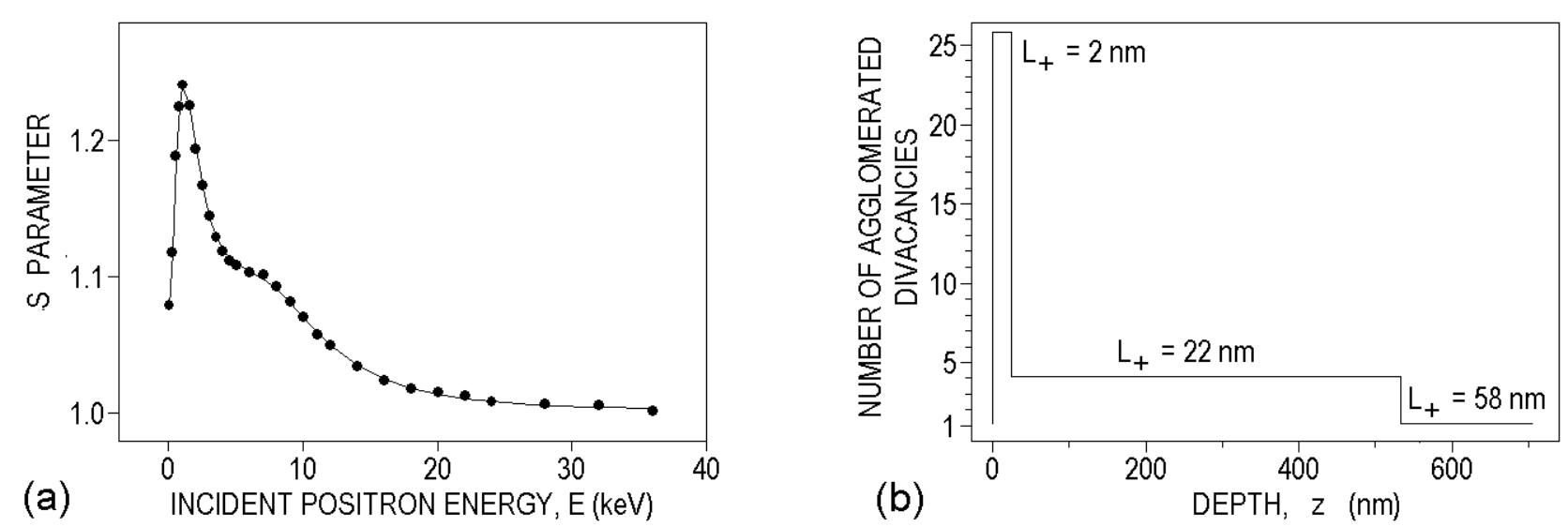

Figure 5. Ion implanted 6H-SiC: (a) $S(E)$ curve (measured data points and fitting curve); (b) layered structure identified from fitting.

solid. Application of this picture may result finally in a more understandable presentation of "size of vacancy-type damage vs. depth" (figure 5) [19]. More details of the data evaluation procedures, and a demonstration of their application to further investigations of ion implantation into $6 \mathrm{H}-\mathrm{SiC}$, can be found elsewhere [20].

For the sake of interpretation and comparison of SPIS results it is also common to discuss $S$ $W$ plots of experimental data as a function of positron energy $E$. When only two distinct annihilation processes, characterized by $\left(S_{1}, W_{1}\right)$ and $\left(S_{2}, W_{2}\right)$, contribute to a set of experimental data, a straight line is obtained in the $S$ - $W$ representation where the endpoints represent the two states themselves. Such a case can, for instance, be a film of a certain thickness on top of a different substrate material. Any deviation from the straight line with increasing $E$ will point to the existence of a further state $\left(S_{3}, W_{3}\right)$ being attractive to positrons, e.g., the "interface" between the film and the substrate. A more sophisticated example of such a plot, based on investigations of the $\mathrm{SiO}_{2} / \mathrm{Si}$ system [21], is illustrated in figure 6.

In principle, an $S$ - $W$ plot could be created for every material or layered system investigated. However, this requires that measurements were taken in exactly the same conditions, namely with a constant energy resolution, so that all data can be related to the same reference value of $S$, i.e. to a

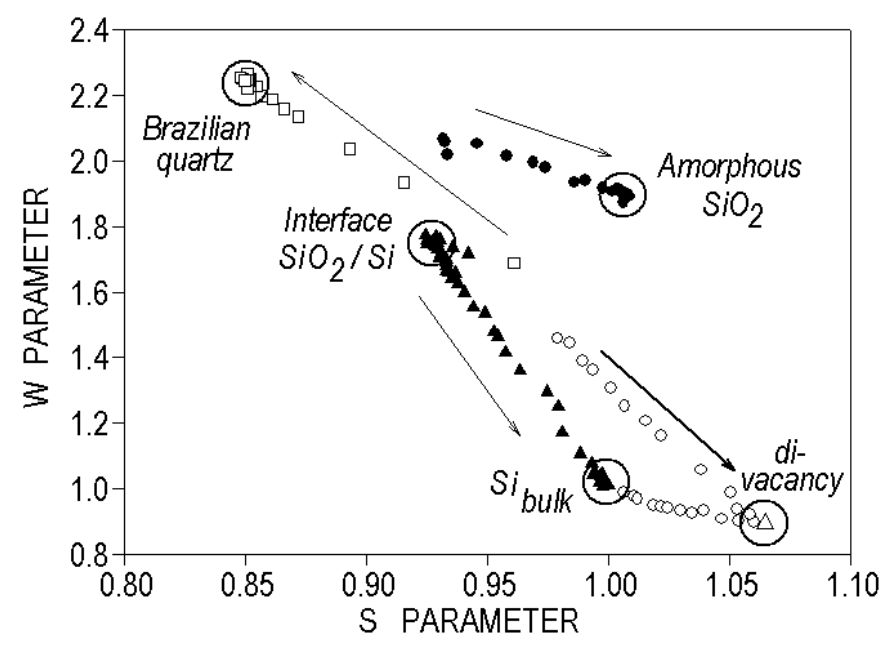

Figure 6. S-W plot of data measured with mono-energetic positrons of increasing energy. The various $\mathrm{SiO}_{2}$ and $\mathrm{Si}$ states, characterized by a typical set of $S$ and $W$ values, are marked by circles. Arrows indicate the increase in chosen positron energy. 
unique reference spectrum. Nonetheless, neglecting small changes in the resolution function within a first order approximation, it may be possible to resort to relative changes of the $S$ parameter to compare various profiles. Therefore, every case investigated needs its own considerations.

\subsection{Investigations of insulating materials: charging effects and limiting factors}

In the course of an investigation of high density polymer films [22], kept electrically isolated in the sample vacuum chamber, a drastic reduction was noticed in the count rate of the $511 \mathrm{keV}$ peak during positron bombardment, more pronounced for low than for high incident energy (figure $7 a$ ). At $0.5 \mathrm{keV}$, for instance, the photopeak area decreased by more than a factor of 4 in less than 2 days. Consequently, up to $5 \mathrm{keV}$ (i.e., below $\sim 500 \mathrm{~nm}$ ), S-profiles were hardly reproducible and strongly depended on the number of days under beam exposure (figure $7 b$ ). Moreover, possibly owing to the high degree of humidity presiding in the environment of UEA Norwich, where these data were actually collected [23], exposing the samples in the air for a couple of minutes before pumping down again was sufficient to completely suppress the charging effect and recover the initial parameter values.
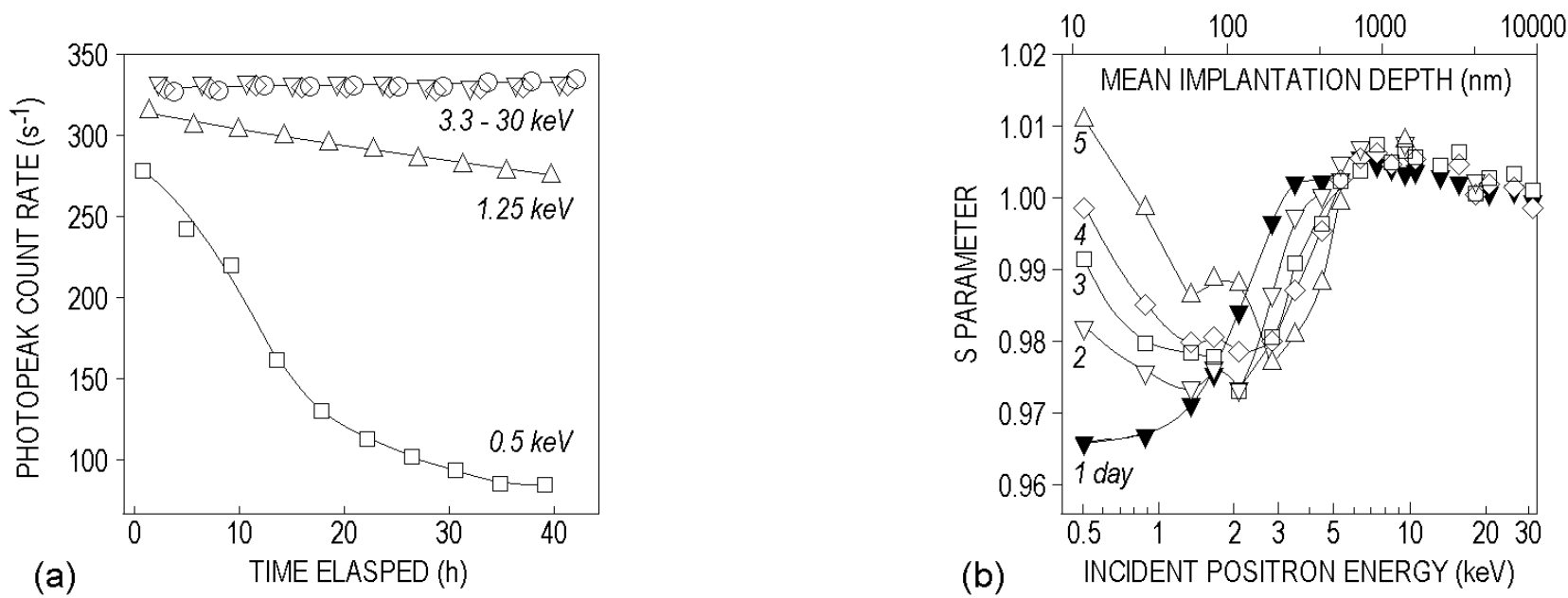

Figure 7. (a) - Time dependence of the photopeak count rate for increasing incident positron energies (in italic). (b) - Time dependence of the positron depth profile for an isolated polymer film (in italic: number of days under beam exposure). Solid lines are guides for the eye.

Since both the total count rate and the peak-to-valley ratio "T" [9] also decreased versus time, and as holding samples in vacuum without beam had no effect, it has been concluded that a subsurface electric field builds up. This causes a drift of thermalized positrons from deeper in the sample towards the surface, where they can form a bound state, so-called positronium (Ps).

A Ps "atom" consists of a positron and an electron, and due to the total spin may exist in an ortho-state (o-Ps, spin state: +1 ) or para-state (p-Ps, spin state: 0 ), subsequently decaying in vacuum into 3 or 2 photons, i.e., with energies below or at $511 \mathrm{keV}$, respectively. Since theory predicts that 3 times as many o-Ps as p-Ps are formed, the parameter "T" may be used as an indicator of Ps formation in a solid. A special branch of surface spectroscopy deals with Ps [1, 8, 24].

Although artefacts may also be observed in other techniques using charged particles, e.g., Rutherford backscattering/channeling (RBS/C), Nuclear Reaction Analysis (NRA), such an effect is surprisingly large in the present study with rather low current (e.g. counting rate). Hence, when performing positron profiling investigations of insulators, the possible occurrence of such artefacts 
should always be kept in mind, and unless special precautions are taken (e.g., carbon deposit on sample surface), the use of high intensity beams for positron profiling may end up to be worthless.

\subsection{Comparison with other techniques}

Of course, there are other techniques in use for the general detection of open volume defects. Several microscopies, like transmission electron microscopy (TEM), atomic force microscopy (AFM), scanning tunnelling microscopy (STM), and optical microscopy (OM), are well known and in practical use in small laboratories. Other defect spectroscopies, like small-angle X-ray or neutron scattering (SAXS, SANS) are also in practical use but have to be operated at larger facilities, like synchrotrons or reactors. Certainly, all these techniques have their specific region of high sensitivity and resolution. Among them, positron analysis (PAS), using either a beam or a sealed source in the classical sandwich configuration, fills a special niche. A comparison of all these methods regarding their sensitivity to defect sizes and concentrations has already been published $[25,26]$.

In figure 8 , based on experimental work referred to in more detail below, such a comparison is given including further particle probe methods, like RBS/C, NRA, and muon spin rotation $(\mu \mathrm{SR})$.

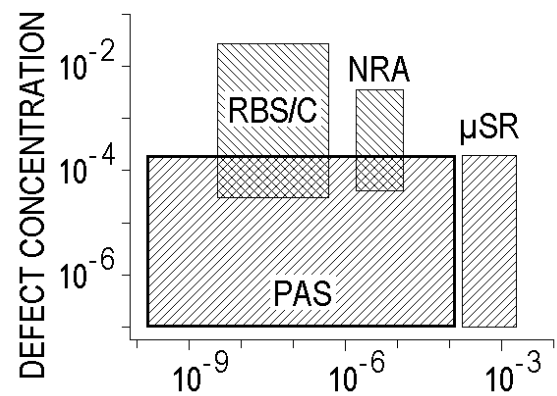

(a)

$$
\text { PROBED DEPTH (meter) }
$$

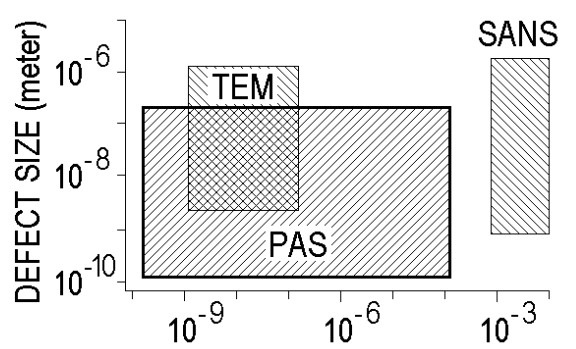

(b)

PROBED DEPTH (meter)

Figure 8. Detection capabilities of defect concentration (a) and size (b) for various microprobe techniques (PAS refers to all positron annihilation techniques).

\section{EXAMPLES OF SUB-SURFACE INVESTIGATIONS}

There is a wide variety of open-volume defects into which positrons may be trapped, including so-called "point defects" such as an isolated (single) vacancy or bound to an impurity, or vacancies clustered together (poly-vacancies) and forming voids when their number becomes large enough, or bubbles if gas atoms or molecules (e.g., helium, hydrogen) are "embedded" therein. Defects, such as linear or edge dislocations or loops as well as dislocation "debris" formed by dislocation tangles, are also candidates for trapping sites. Other "extended" defects can also play a role (phase or grain boundaries, precipitates) if the density of the disturbed surface area per unit volume is large enough so that the average distance between boundaries is comparable to the mean free path of thermalized positron. All these defects may be generated by irradiation, quenching, or deformation resulting from stresses either applied externally (e.g., cold-working), or induced by internal changes in the solid (e.g., phase transition, thermal cycling, precipitation...).

Besides trapping at open-volume defects, a difference in the chemical affinity of the positron for atoms of different nature may either attract the probe to, or repulse it from, particular sites of localization [27]. The change in re-emission properties with increasing annealing temperature, observed in figure $3 b$ for W-Ni alloys, which evolves from a nickel-like to a tungsten-like behaviour, most likely proceeds from such an affinity effect. Nevertheless, a chemical affinity effect 
may easily be confused with concomitant trapping effects due to open volume defects. Coincidence Doppler broadening (CDB) is therefore a more appropriate technique to study affinity effects [28].

It is not the purpose of this review to present a comprehensive outlook of all possible effects which can be investigated by depth profiling. In contrast, this section focuses on some typical damage modes occurring during industrial processes and on applications involving thin layered materials.

\subsection{Studies of stresses induced defects and phase transition in tungsten deposits}

It is common knowledge that in-service properties of metallic materials, under mechanical stresses or in aggressive chemical environments, strongly depend on the state of the exposed surface, where cracks or corrosion pits may be initiated, before progressing into the bulk. Whereas damaging within the first couple of microns is usually acceptable because it does not significantly affect bulk properties, such tolerance is not allowed for thin layers. In this latter case, nondestructive and easy-to-handle techniques, such as X-ray diffraction (XRD) for instance, cannot assess the detailed microstructure within a surface layer thinner than $\sim 0.1 \mu \mathrm{m}$.

In order to demonstrate the ability of SPIS to tackle such problems [29], depth profiling was performed on submicron layers of tungsten deposited by physical vapour deposition on hard steel, before and after bending tests (figure $9 a$ ).
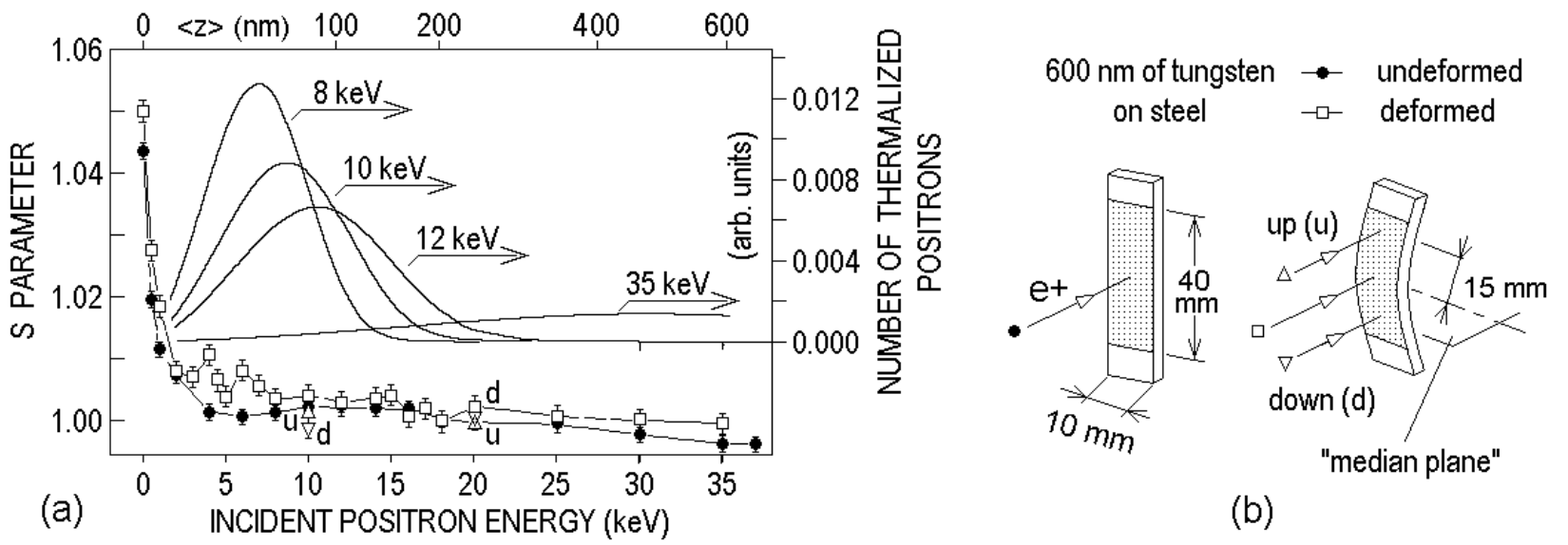

(b)

Figure 9. a) $S$ parameter depth-dependence in tungsten deposit on steel substrate before (closed circles) and after bending (open symbols). The $S$ scale is normalized to the bulk value, at around 20 $\mathrm{keV}$. The upper scale and the right hand scale refer respectively to the mean positron depth $<_{z}>$, and to Makhov distributions of thermalized positrons for typical incident energies in tungsten. b) Sample dimensions and beam spot impacts. Lines joining symbols are only eye guides.

Before bending, the $S$ profile exhibits a smooth dependence on positron incident energy, indicating that the tungsten layer is homogeneous. Indeed, grazing incidence XRD measurements reveal a "pure" body-centred cubic $\alpha$-phase over a mean depth of $\sim 200 \mathrm{~nm}$. Below $3.5 \mathrm{keV}$ (i.e., for $<z>\leq 15 \mathrm{~nm}$ ), the rise of $\mathrm{S}$ towards the surface, is possibly due to either a surface pollution (actually observed in most of our experiments, owing to the rather "poor" vacuum in the beam setup), or/and to a transition from the $\alpha$-phase to the $\beta$-phase, the latter being associated to a higher oxygen solubility than the former.

Bending provokes an obvious scattering trend to higher levels of $S$ within the first $80 \mathrm{~nm}$ range $(\sim 10 \mathrm{keV})$. This effect only happens around the bent area, as demonstrated by the $S$ profile, which remained unchanged when measured above (up) or below (down) the bending median plane of the sample, using a positron spot size of $\sim 4 \mathrm{~mm}$ (figure $9 \mathrm{~b}$ ). This change possibly results from 
defects produced during the emergence of the $\beta-\mathrm{W}$ phase in the near-surface region, as the $\alpha \rightarrow \beta$ phase transition is known to be favoured by a compressive-to-tensile stress transition [30].

The above measurements were complemented with depth profiling and grazing angle XRD, performed on a set of tungsten films deposited in various sputtering conditions on KAPTON ${ }^{\circledR}$ [31]. In order to avoid charging effect artefacts due to a lasting positron beam exposure (see section 2.5 ), the metal surface was grounded. In figure $10 a$ and $b, S$ profiles exhibit a marked difference predominantly related to the final thickness of the deposit. Moreover, an overall increase of $S$ seems to result in a decrease in the $\alpha-\mathrm{W}$ mean content (i.e., in a correlative increase in the $\beta-\mathrm{W}$ content), which in turn also appears to follow a decrease in the working gas pressure. Such behaviour seems to validate the above interpretation of bending test results. However, no clear picture can yet be drawn to explain the erratic fine structure of the depth dependence of $S$, which indicates a lack of homogeneity in the W-KAPTON samples, as opposed to the W-steel samples.
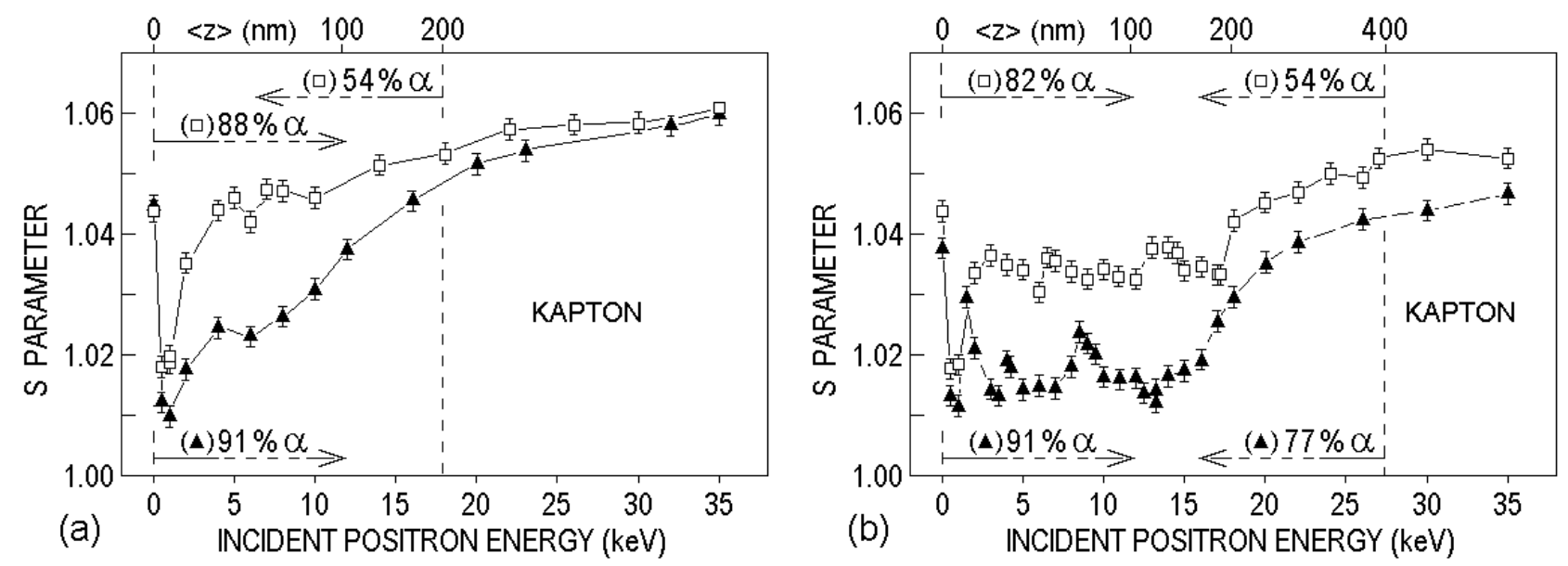

Figure 10. $S$ depth-dependence in $200 \mathrm{~nm}$ (a) and $400 \mathrm{~nm}$ (b) thick tungsten deposits on KAPTON substra-tes, under various conditions. Open and closed symbols correspond to low and high working gas pressure, respectively. For each sample, concentrations of $\alpha$-phase are measured by grazing XRD from either the front side $(\rightarrow)$ or from the back side $(\leftarrow)$ (i.e., through the KAPTON in the latter case). The reference value for $S$ and upper scales are the same as in figure $9 a$. Lines joining symbols are only eye guides.

Actually, irrespective of the hesitation to draw detailed correlations between $S$ and the parameters controlling the sputtering process, the prime purpose of this latter study was to develop a SPIS blind test to check the uniformity of stress (or phase) distribution within the deposit. In this respect, the present measurements indeed demonstrate that SPIS is a unique technique to study the homogeneity of internal stresses and phase transitions in thin layers.

\subsection{Other examples of bilayer investigations (polymer/metal interfaces)}

In this section two examples of bilayer investigations for industrial applications are reported, which deal with proprietary research and development (R\&D). Hence, very little information can be disclosed about the characteristics of the samples, except that some of them met technological specifications while others departed from these requirements.

The scheme was to compare positron depth profiles measured on samples selected as references among a production set, for they successfully passed relevant screening tests, with profiles taken on other samples chosen randomly, in hope that such a comparison could serve as an appropriate quality control, and avoid further tedious characterizations. 
In the first example, a series of aluminium coated films of polymers were selected among 3 distinct categories exhibiting good, poor or quasi inexistent adhesion, respectively, owing to different surface treatments prior to metallization. Using the UEA-Norwich beam [23], SPIS was performed before and after coating, resulting in highly reproducible profiles for similar samples pertaining to a given category (figure 11a). Before coating, no clear cut differences are observed between the profiles, except that an overall high level for $S$ corresponds to polymer-bearing surfaces yielding no adhesion. Irrespective of the polymer pre-treatment, a coating of $20 \mathrm{~nm}$ aluminium drastically increases the $S$ parameter near the surface, whereas a constant value is reached in the polymer bulk, i.e., above $\sim 2 \mu \mathrm{m}$. Inferring that the maximum of $S$ and the dip that follows, within the first $20 \mathrm{~nm}$, correspond to positrons sensing the metal-polymer interface, it can be concluded that the more the interface appears to extend into the polymer, the lesser the adhesion. In other words, a narrow interface seems to secure good adhesion.

Although such an investigation remains in a preliminary stage from a scientific point of view, it shows the obvious benefit that SPIS could offer in on- or off-line quality control at an industrial production site, for instance before the manufacture and testing of end-product devices made from metal-coated polymer produced in huge length films. Jean et al. [32] have also shown that the technique applies to a wide variety of cases.
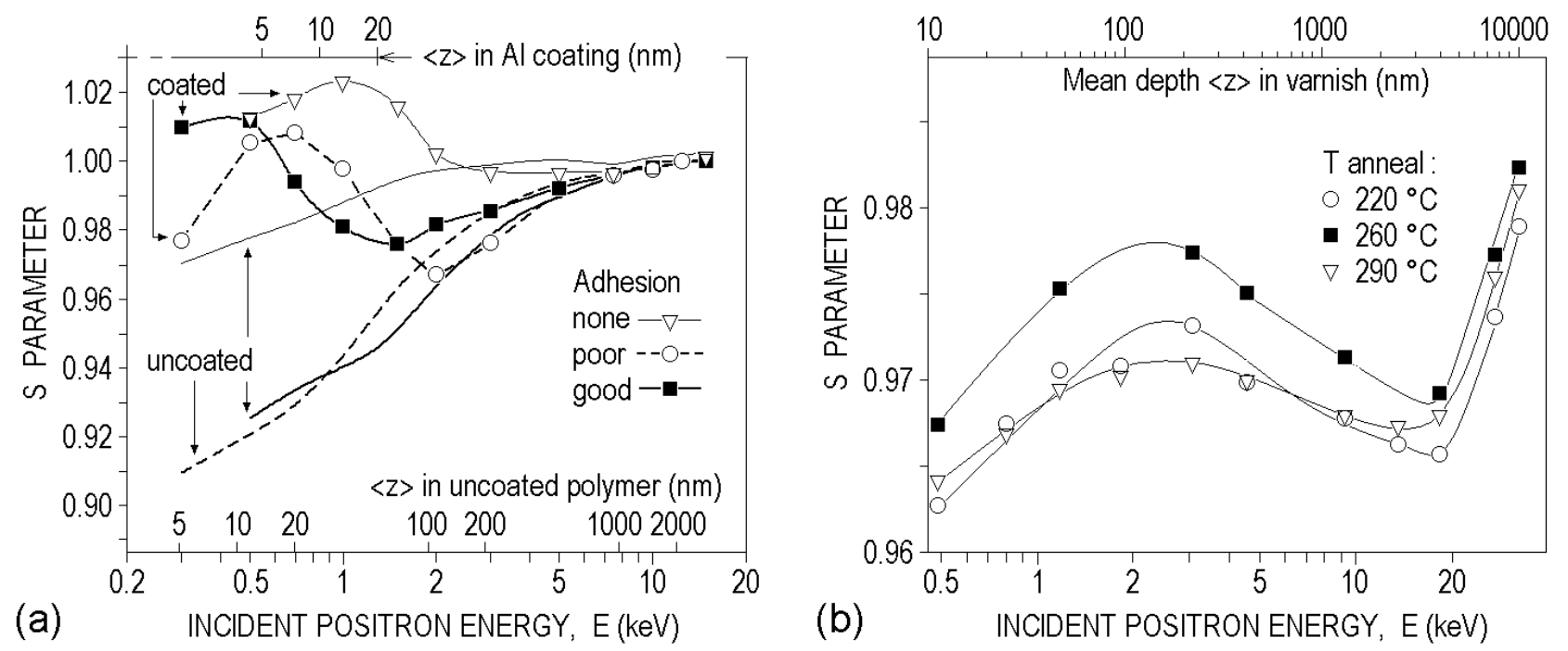

Figure 11. a) Positron depth profiling in polymers with and without aluminium coating. b) Positron depth profile dependence on heat treatment of varnish layers protecting aluminium. The reference for $S$ values is the bulk value of figure 11a. Lines are guides for the eye.

In a second example of industrial application, where a polymer is assumed to protect a metal, (e.g. aluminium), the influence of heat treatment has been monitored. Here again, while coated samples of identical origin were known to have undergone different treatments, the nature of the protecting varnish could not be disclosed. In this case, the varnish-metal bonding (with an interface at around $\sim 2 \mu \mathrm{m}$ ) was conversely examined from the polymer side in a series of SPIS "blind" tests (figure 11b). Profiles exhibit a marked difference in the $S$ depth dependence only for the sample treated at intermediate temperature. Strikingly, this temperature, which induces the highest $S$ maximum, in the $100 \mathrm{~nm}$ range, corresponds to the annealing that ensures the best anti-corrosion protection of this varnish-aluminium bonding. Since positron profiling lasts only a few hours, as opposed to several months for relevant corrosion tests, it clearly shows once more how much industrial R\&D on protective coatings can profit from SPIS. Likewise, Hulett et al. [33] have demonstrated how positron probing could monitor the effects of weathering on paints. 


\subsection{Surface modification of materials by ion implantation}

Beam processing in industrial applications primarily concerns surface treatment of solids [9], while in other special studies ion implantation is designed to mimic long lasting irradiation occurring (e.g. during storage) in ceramics confining nuclear waste [34] or in the fissile material itself $[35,36]$. The ion energy is thus mostly limited to the sub-MeV range in the former case, whereas in the latter, it seldom exceeds a few $\mathrm{MeV}$, to prevent radio-activation of the material. Therefore, in either case, whatever the ion mass, the affected depth of the solid never extends beyond a few microns. In order to assess the intended or expected structural modifications, it is therefore of paramount importance to have access to a probing technique that matches the same depth range. SPIS is indeed the appropriate non destructive tool for such an assessment.
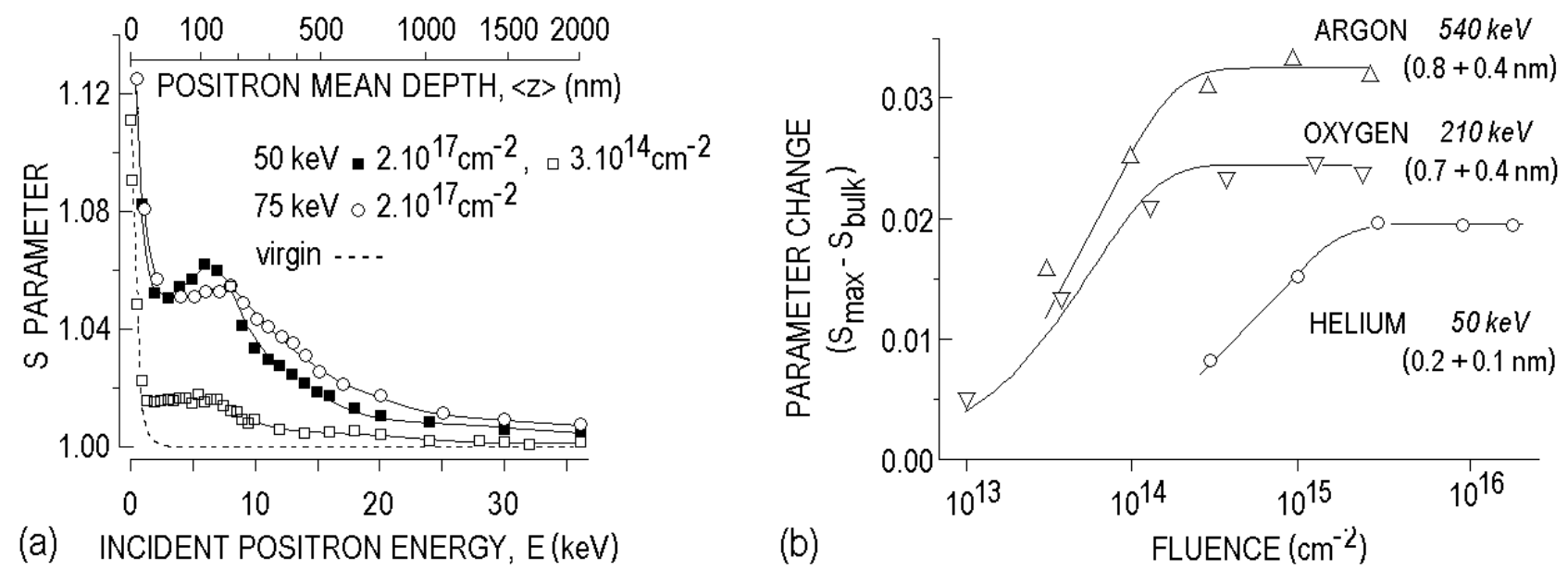

Figure 12. a) Positron depth profiling in $\mathrm{YSZ}$ cubic single crystals $\left(9.5 \% \mathrm{Y}_{2} \mathrm{O}_{3}\right)$ before and after implantation with 50 and $75 \mathrm{keV}$ He-ions at various fluences. $S$ profiles are fitted with VEPFIT [18]. b) Fitted fluence dependence of the $S$-maximum induced by implantation of $\mathrm{He}$, Ar and O ions at given energies (italic numbers). The radius between brackets corresponds to the defect mean size at the saturation trapping (plateau) for the given implantation.

Yttria stabilized zirconia (YSZ), which crystallizes in the cubic phase at room temperature for doping in the range of $\sim 10 \% \mathrm{Y}_{2} \mathrm{O}_{3}$, is a convenient material to gain knowledge of the various parameters controlling structural damage induced by ion implantation. Indeed, owing to its chemical and thermal stability (melting point $\approx 3000 \mathrm{~K}$ ), and its ability to grow into large single crystals, suitable for a wide variety of techniques, YSZ has been studied extensively during the last decade, using various positive probes [26]. These investigations have been complemented by SPIS investigations [37] aimed at unraveling the interconnected dependence of energy and fluence of bombarding ions on the damage structure.

In spite of the presence of a large quantity of structural oxygen vacancies (a few \%) resulting from the presence of the trivalent stabilizing cation $\mathrm{Y}^{3+}$, the positron profiling unveiled a maximum of $S$ after ion implantation (as evidenced in figure 12a) for ${ }^{4} \mathrm{He}$ ions of various energies and fluences. Incidentally, due to a possible significant ionic mobility in YZS, no charging effect seemed to occur, since $S$ profiles were not affected when samples were carbon-coated with a few $\mathrm{nm}$ to prevent any subsurface field build up as reported in section 2.5.

Although the Doppler broadening analysis could not, as in some other cases, achieve a proper identification of the nature of the damage produced by this implantation, it could be nevertheless asserted that the type of these defects was different from the native ones. Moreover, for 
low fluences, assuming that defect cascades generated by single ion impacts did not overlap, a model could fit the $S$ dependence on fluence and, as trapping reached saturation, a mean radius of the defect derived (figure 12b), which seemed to depend slightly on the ion mass.

Applying the TRIM code [38] to perform Monte-Carlo simulations of the ion bombardment effect revealed a striking feature (figure 13a), namely: the peak location of the vacancy distribution did not match - by a large margin - the mean depth of the $S$ peak where positrons were supposed to undergo maximum trapping. Actually, for many more ions, with energies chosen to fit in a depth range below $200 \mathrm{~nm}$, a ratio of $\sim 0.5$ could be found between the location of the $S$-peak and the associated ion projected range $R_{\mathrm{p}}$ (figure $13 \mathrm{~b}$ ).

This so-called $R_{\mathrm{p}} / 2$ effect, already observed in other materials [39, 40], arises from an incomplete cancellation, during cascade rearrangement, of interstitial and vacancy shifted-apart distributions. It has to be accounted for when ion beams are used to manufacture microelectronic chips, such as for instance in the so-called Smart Cut ${ }^{\circledR}$ process (or "ion cut") [41], based on the build up of a sub-surface damage layer where cleavage can take place in order to remove a thin layer from its parent wafer. This $R_{\mathrm{p}} / 2$ effect also appears instrumental in the following example of multilayer processing.
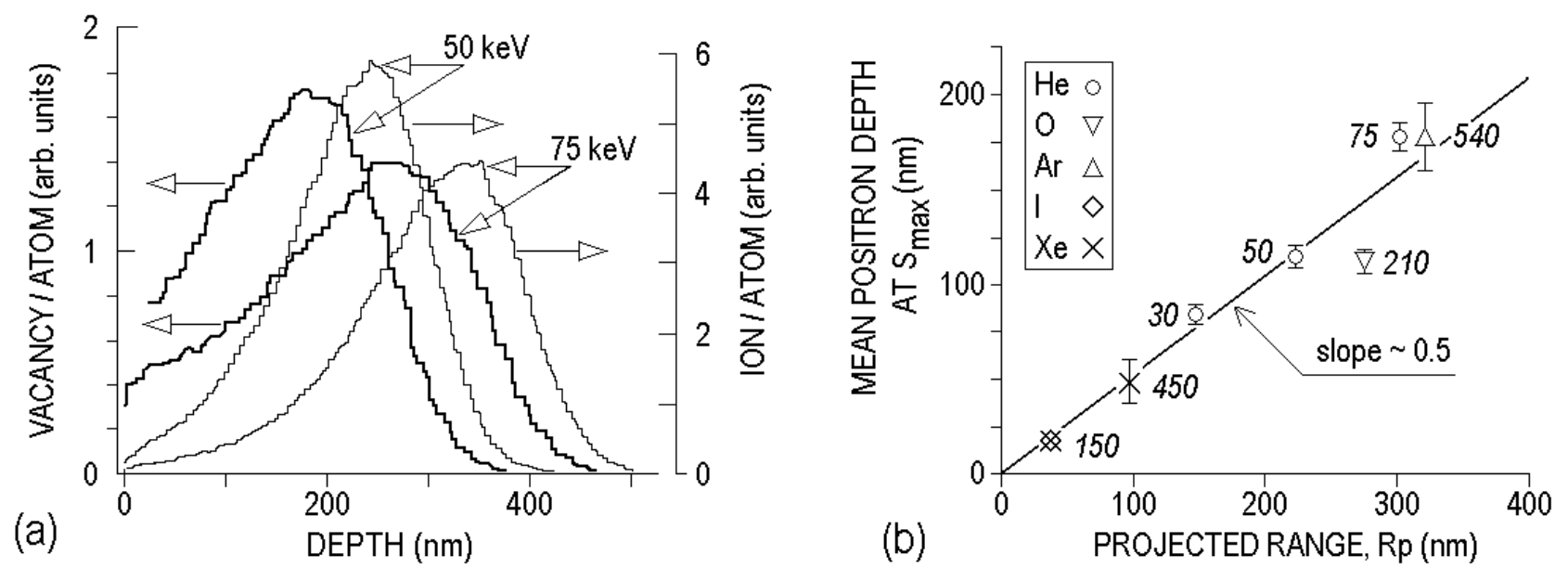

Figure 13. Ion implantation in YSZ (density $\left.=5.92 \mathrm{~g} \mathrm{~cm}^{-3}\right)$. a) TRIM simulation of helium ion distributions (thin lines) and associated vacancy distributions (thick lines). b) $S$-peak mean depth versus. $\mathrm{R}_{\mathrm{p}}$ for various implanted ions. Italic numbers refer to ion energies in $\mathrm{keV}$ (slope $=0.53$ with $\left.\mathrm{r}^{2}=0.95\right)$.

\subsection{Multilayered materials}

New integrated circuits featuring complex patterns, with sizes smaller than $100 \mathrm{~nm}$, are nowadays printed by means of extreme-ultraviolet (EUV) lithography [42, 43]. In this process, the low normal-incidence reflectivity exhibited in the soft X-ray wavelength region by standard optical materials, can be enhanced by surface coating with multilayers, owing to constructive interference occurring in this stack. Multiple Mo/Si bilayers, with "elemental" thickness in the range of $\sim 3$ to $\sim 20 \mathrm{~nm}$, have proven to be a suitable technological option to build such optical devices. Moreover, further improvement of the reflectivity of these multilayers, mainly limited by interface roughness, may be achieved by ion-implantation (e.g., argon or oxygen) [42, 44] which tends to smooth the layer boundaries.

In an attempt to monitor the effect of various conditions of ion-implantation on the interface roughness, i.e. on the native defects therein, positron depth profiling has been performed on a series of $\mathrm{Mo} / \mathrm{Si}$ multilayers deposited on silicon (figure 14a). As expected, due to the positron energy 
straggling exemplified in figure $14 \mathrm{~b}$, and in figure $1 \mathrm{~b}$, for an average density twice as low, there was no hope to single out individual layers or interfaces.

Nevertheless, a drastic change in the depth dependence of the $S$ parameter was observed with increasing oxygen fluence. From figure $13 \mathrm{~b}$, derived for a material of density equivalent to the average density of Mo/Si bilayers $\left(\sim 6 \mathrm{~g} . \mathrm{cm}^{-3}\right)$, it may be inferred that, for $180 \mathrm{keV}$ O-ions, the resulting $S$ profile representing the distribution of implantation-induced defect should be peaking at around $100 \mathrm{~nm}$. In contrast, we observe a dip, as if the net effect of these new defects had been to help the native defects located at interfaces to anneal out. Indeed, reflectivity measurements indicate a "densification" of the layered material after implantation [44].
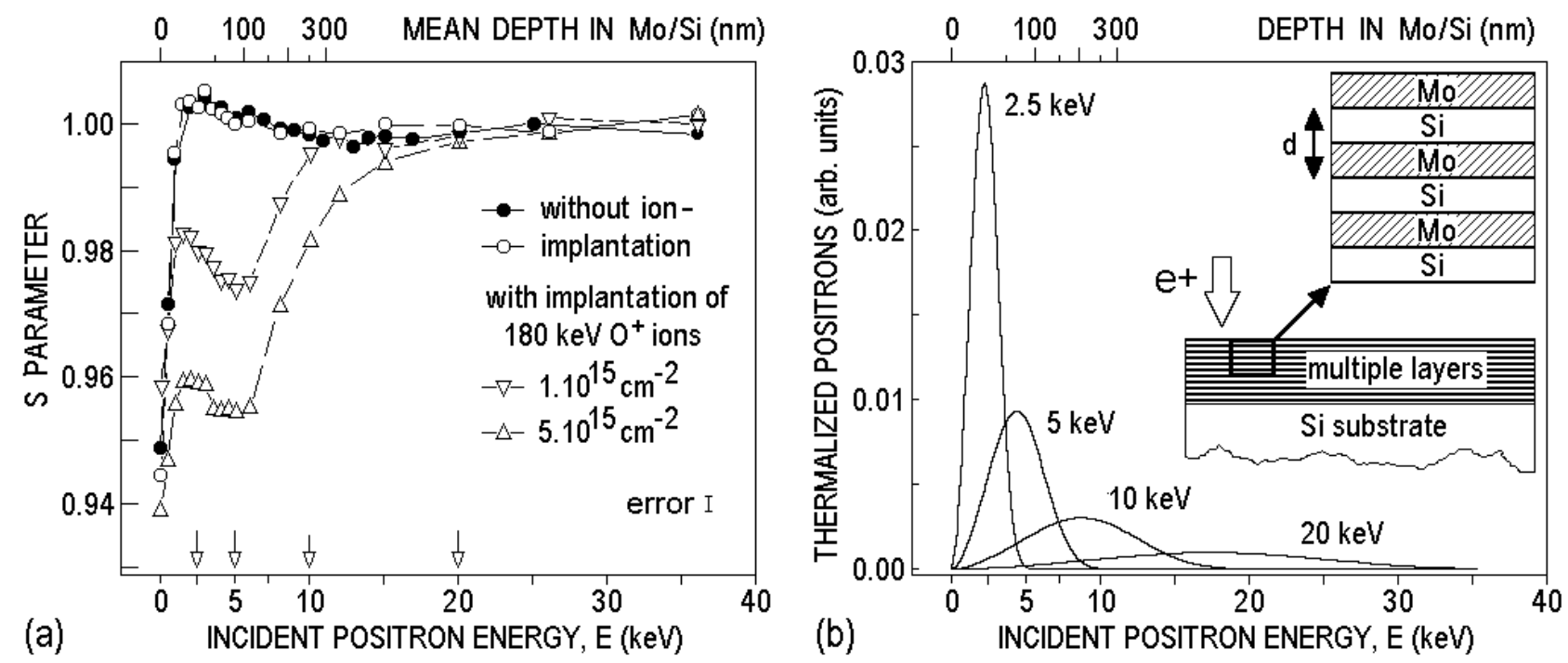

Figure 14. Periodic multilayer samples of Mo/Si deposited on Si substrate without and with subsequent $180 \mathrm{keV} \mathrm{O-ions} \mathrm{implantation} \mathrm{at} \mathrm{various} \mathrm{fluences} \mathrm{(} 40 \mathrm{Mo} / \mathrm{Si}$ bilayers extend over a depth of $\sim 300 \mathrm{~nm}$; the mean depth $(<\mathrm{z}>)$ is calculated for a bilayer average density of $\left.6 \mathrm{~g} . \mathrm{cm}^{-3}\right)$. a) $S$ dependence on positron incident energy. Notice the profile reproducibility for the two non-implanted samples. b) Corresponding distribution of thermalized positrons for 4 typical incident energies $\left(\mathrm{d}=\mathrm{d}_{\mathrm{Mo}}+\mathrm{d}_{\mathrm{Si}}\right.$, with $\mathrm{d}_{\mathrm{Mo}} \sim 3 \mathrm{~nm}$ and $\mathrm{d}_{\mathrm{Si}} \sim 4 \mathrm{~nm}$ comprising interface mean widths $\Delta \mathrm{d}(\mathrm{Mo}-\mathrm{Si}) \leq 0.3 \mathrm{~nm}$ and $\Delta \mathrm{d}(\mathrm{Si}-\mathrm{Mo}) \leq 0.2 \mathrm{~nm}$, respectively).

Hence, whereas SPIS does lack of precision to "visualize" single layers or interfaces of nanoscale widths, it proves to be a useful tool to monitor desirable technological and structural properties of these soft X-ray mirrors.

\section{GENERAL DISCUSSION, CONCLUSIONS AND OUTLOOK}

Examples of studies reported in the above sections show that, whereas slow positron implantation spectroscopy has already been implemented in a broad range of research topics, it can also be effectively used for routine quality control in industrial processes.

Low-energy positron beams can be rather easily built, especially for Doppler broadening measurements, which only need a continuous flux. Indeed, most of the beams currently available in Europe, and used for materials characterization (figure 15a), operate on a direct-current mode. Their positron primary source - i.e. before energy moderation - usually relies i) on the steady emission from a long lived isotope, mainly commercially-available ${ }^{22} \mathrm{Na}$, or ii) on the on-line production of either short lived isotopes or direct photo-production via Bremsstrahlung, depending on the nuclear or particle physics facility hosting the beam. 
Some limitations have also been pointed out such as possible artefacts relevant to probing with charged particles or those related to energy straggling of the positron. Indeed, investigation of ultra-thin multilayers, for instance, can be a challenge. Moreover, given the uncertainties indicated in figure $15 b$ for typical energy values, it is obviously worthless to use positron energies larger than $30-40 \mathrm{keV}$ for the characterization of submicron thin layers or layers located at larger depths than $1 \mu \mathrm{m}$. To overcome limitations associated to any required deep layer profiling, destructive thinning procedures have to be applied instead, in order to achieve a reasonably good depth resolution. A demonstration of such an investigation can be found in the literature [39]. However, besides losing the non-destructive feature of the SPIS method, substantial efforts are required to reach the intended result.

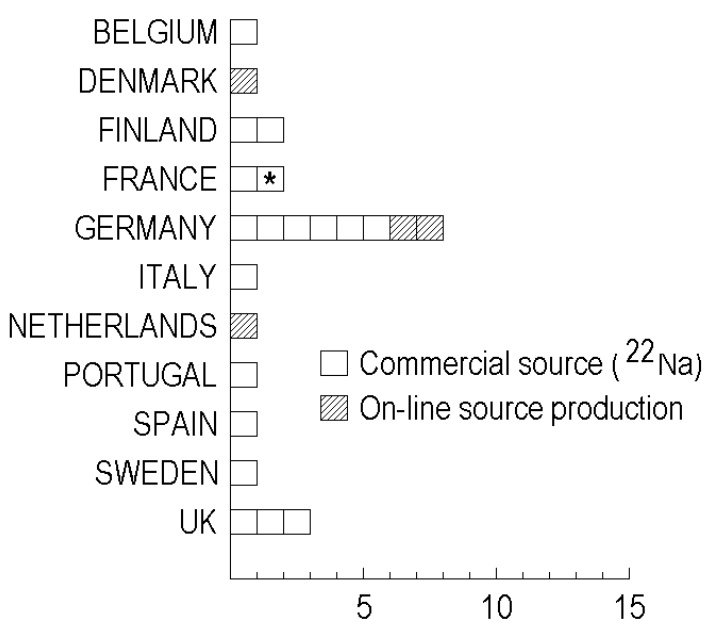

(a) NUMBER OF POSITRON BEAMS IN THE E.U.

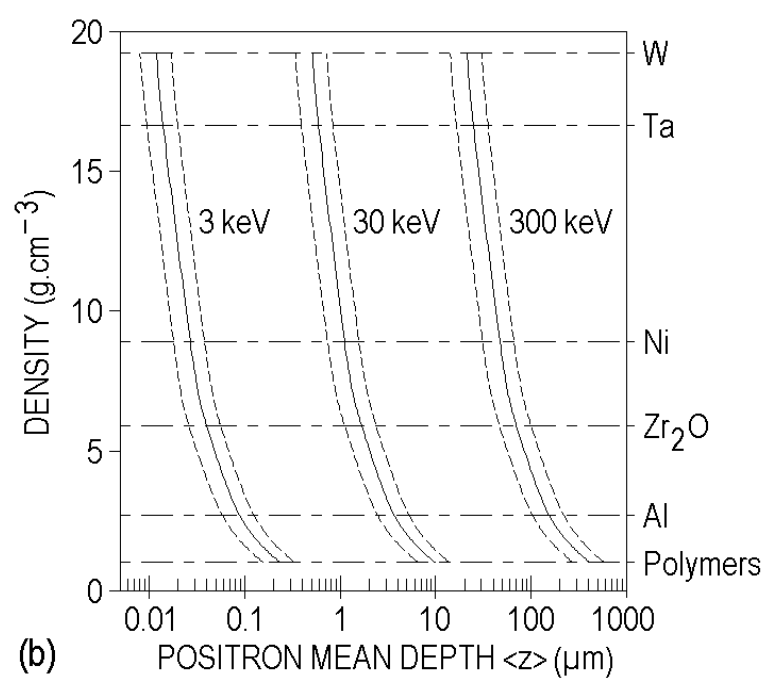

(b)

Figure 15. a) Updated, but possibly not exhaustive, list of low energy positron beams in Europe (*: French-Swiss joint venture at CERN [47]). b) Mean penetration depth (logarithmic scale) and uncertainty limits due to energy straggling (dashed curves) in various dense solids for three typical energies of incident positrons

Table I. Tracking of open-volume defects (nanoporosity) in solids. Examples refer to a density $\sim 1$ g. $\mathrm{cm}^{-3}$ (Lifetime technique based on secondary electron detection is not considered).

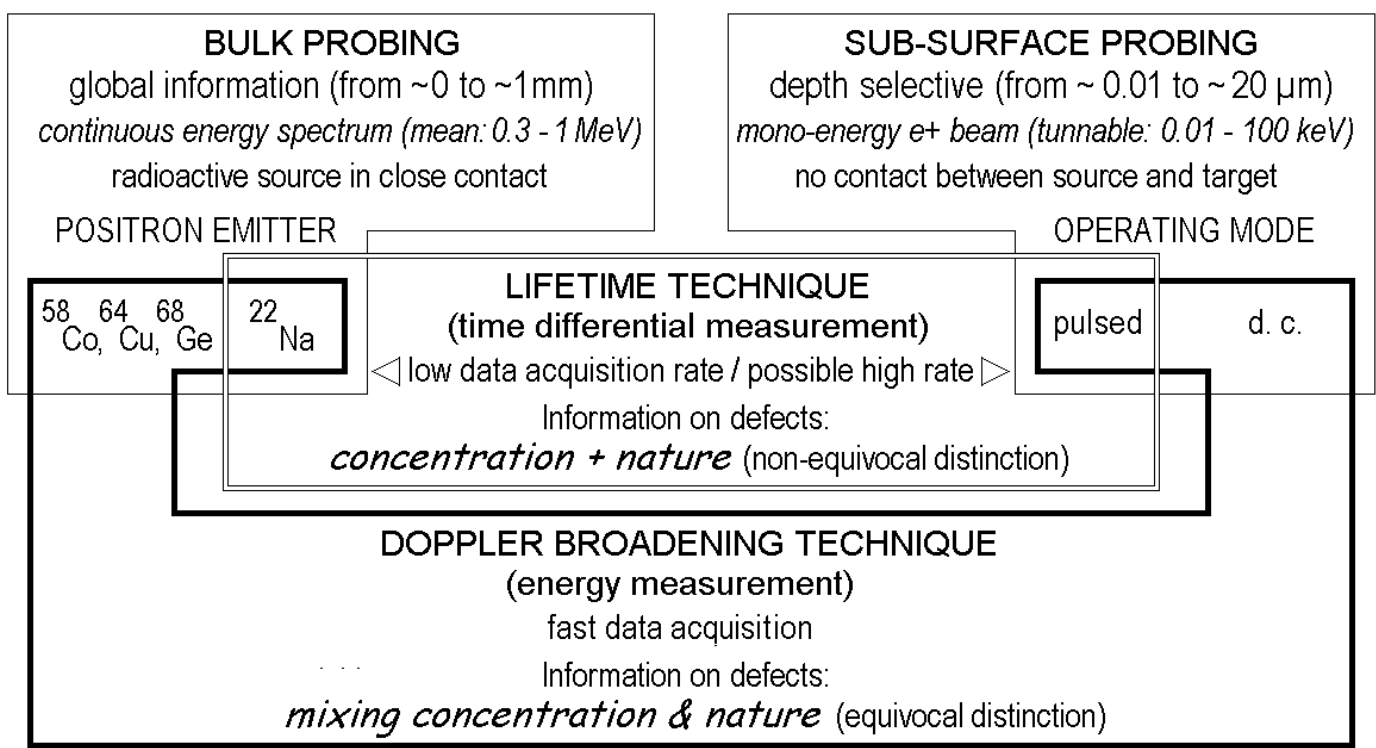


Doppler broadening analysis has also its own limitation, since, in most cases, it can only provide global information on existing defects as opposed to time differential measurements of the positron in the sample before annihilation. However, this latter lifetime technique requires more sophisticated beam setups, where a clock starting signal relies on either i) a pulsed positron flux formed by combination of chopping and bunching elements [45-47] or ii) the emission of secondary electrons induced by positron impinging the target [48].

Table I summarizes the main features, for both bulk and sub-surface measurements, to be considered when a certain positron method has to be selected for materials analysis. In connection with circumstances of availability/access at given research locations in Europe, and worldwide, or special technical needs, positron-emitting isotopes other than ${ }^{22} \mathrm{Na}$ have been used in materials science and are also mentioned in Table $I$. These comprise ${ }^{58} \mathrm{Co}(71 \mathrm{~d} ; 0.5 \mathrm{MeV}),{ }^{64} \mathrm{Cu}(12.7 \mathrm{~h}$; $0.7 \mathrm{MeV}$ ), and ${ }^{68} \mathrm{Ge}(271 \mathrm{~d}, 1.9 \mathrm{MeV})$, with given (half-life; $\sim E_{\max }$ ) values. Especially, their corresponding $E_{\max }$ values have to be considered in order to calculate the needed sample thicknesses for bulk studies, and the corresponding design of a moderator to get mono-energetic positrons.

Only very recently, a so-called "Depth-selective positron nano-porosimetry system PALS-1" became commercially available [49]. This fact might help to further promote the application of this method for industrial R\&D. However, it should be emphasized that all positron methods are always complementary to other useful methods; the concerted application of different methods may finally be the best solution to solve any given problem in materials science.

\section{ACKNOWLEDGMENTS}

One of the authors (R.I.G) is deeply indebted to the French Department of Defence (DGA) for lasting support, and he acknowledges IBS-France for providing valuable multilayer samples.

\section{REFERENCES}

[1] A. Dupasquier, A.P. Mills, Jr, Positron Spectroscopy of Solids. IOS, Amsterdam (1995).

[2] R. Krause-Rehberg, H.S. Leipner, Positron Annihilation in Semiconductors - Defect Studies. Springer, Berlin (1999).

[3] G. Brauer, Positronen Annihilation Spektroskopie, in: Werkstoff-analytische Verfahren - Eine Auswahl. H-J. Hunger ed., Deutscher Verlag für Grundstoffindustrie, Leipzig, (1995) 524550 .

[4] R.M. Nieminen, J. Oliva, Theory of positronium formation and positron emission at metal surfaces, Phys. Rev. B 22 (1980) 2226-2247.

[5] S. Valkealahti, R.M. Nieminen, Monte Carlo calculations of $\mathrm{keV}$ electron and positron slowing down in solids II, Appl. Phys. A 35 (1984) 51-59.

[6] A. Vehanen, K. Saarinen, P. Hautojärvi, H. Huomo, Profiling multilayer structures with monoenergetic positrons. Phys. Rev. B 35 (1987) 4606-4610.

[7] H. Schut, private communication (1996).

[8] P.J. Schultz, K.G. Lynn, Interaction of positron beams with surfaces, thin films, and interfaces, Rev. Modern Phys. 60 (1988) 701-779.

[9] P.G. Coleman, Positron beams and their applications. World Scientific, Singapore, (2000).

[10] A.P. Mills, Jr., E.M. Gullikson, Solid neon moderator for producing slow positrons, Appl. Phys. Lett. 49 (1986) 1121-1123.

[11] K.G. Lynn, B.T.A. McKee, Some investigations of moderators for slow positron beams, Appl. Phys. 19 (1979) 247-255. 
[12] C.D. Beling, R.I. Simpson, M. Charlton, F.M. Jakobsen, T.C. Griffith, P. Moriarty, S. Fung, A field-assisted moderator for low-energy positron beams, Appl. Phys. A 42 (1987) 111-116.

[13] C.D. Beling, R.I. Simpson, M.G. Stewart, Y.Y. Wang, S. Fung, J.C.H. Wai, T.N. Sun, Positron mobility measurements and their relevance to defect and impurity studies in semiconductors and insulators, Phys. Stat. Sol. 102 (1987) 537-547.

[14] J. Störmer, A. Goodyear, W. Anwand, G. Brauer, P.G. Coleman, W. Triftshäuser, Silicon carbide: A new positron moderator, J. Phys.: Condens. Matter 8 (1996) L89-L94

[15] R.I. Grynszpan, P.G. Coleman, Positron re-emission from electro-deposited NiW, Meas. Sci. Technol. 12 (2001) 163-166.

[16] P.G. Coleman, Positron microscopes and microprobes. In: Positron beams and their applications, P.G. Coleman, ed., World Scientific, Singapore, (2000) 227-236.

[17] A.H. Weiss, P.G. Coleman, Surface science with positrons. In: Positron beams and their applications, P.G. Coleman, ed., World Scientific, Singapore, (2000) 129-189.

[18] A. van Veen, H. Schut, J. de Vries, R.A. Hakvoort, M.R. Ijpma, Analysis of positron profiling data by means of "VEPFIT". In: Positron beams for solids and surfaces, P.J. Schultz, G.R. Massoumi, P.J. Simpson editors. Amer. Inst. Phys., NY (1990) 171-196.

[19] W. Anwand, G. Brauer, W. Skorupa, Evolution of ion implantation-caused vacancy-type defects in $6 \mathrm{H}-\mathrm{SiC}$ probed by slow positron implantation spectroscopy, Appl. Surf. Sci. 184 (2001) 247-251.

[20] W. Anwand, G. Brauer, W. Skorupa, Vacancy-type defects in $6 \mathrm{H}-\mathrm{SiC}$ caused by $\mathrm{N}^{+}$and $\mathrm{Al}^{+}$ high fluence co-implantation, Appl. Surf. Sci. 194 (2002) 131-135.

[21] G. Brauer, W. Anwand, W. Skorupa, A.G. Revesz, J. Kuriplach, Characterization of the $\mathrm{SiO}_{2} / \mathrm{Si}$ interface by positron annihilation spectroscopy, Phys. Rev. B 66 (2002) 195331-195341.

[22] P.G. Coleman, S. Kuna, R.I. Grynszpan, Slow positron implantation spectroscopy of insulators: charging effects, Materials Sci. Forum 255 (1997) 668-670.

[23] N.B. Chilton, P.G. Coleman, A computer-controlled system for slow positron implantation spectroscopy, Meas. Sci. Technol. 6 (1995) 53-59.

[24] M. Charlton, G. Laricchia, Positron impact phenomena, J. Phys. B: At. Mol. Opt. Phys. 23 (1990) 1045-1078.

[25] R.H. Howell, T.E. Cowan, J. Hartley, P. Sterne, B. Brown, Positron beam lifetime spectroscopy of atomic scale defect distributions in bulk and microscopic volumes, Appl. Surf. Sci. 116 (1997) 7-12.

[26] R.I. Grynszpan, G. Brauer, W. Anwand, L. Malaquin, S. Saudé, I. Vickridge, E. Briand, Radiation damage in zirconia investigated by positively charged particles, Nucl. Instr. and Methods (2007), in press, and references therein.

[27] D.M. Hunter, R.I. Grynszpan, A.S. Arrott, Search for positron localization near transitionmetal solutes of negative effective charge in Ni and Cu, J. de Physique VI 3 (1993) 1987-1992

[28] K.G. Lynn, J.R . MacDonald, R.A. Boie, L.C. Feldman, J.D. Gabbe, M.F. Robbins, E. Bonderup, J. Golovchenko, Positron-Annihilation Momentum Profiles in Al: Core contribution and the independent-particle model, Phys. Rev. Lett. 38 (1977) 241-244.

[29] R.I. Grynszpan, G. Galant, Quand l'antimatière sonde la matière, Actualités des Arts et Métiers 250 (2001) 23-25.

[30] K. Bouziane, M. Manor, F. Meyer, DC magnetron sputtered tungsten : W film properties and electrical properties of W/Si Schottky diodes, Appl. Phys. A 81 (2005) 209-215.

[31] J. Ligot, S. Benayoun, J.J. Hantzpergue, J.C. Remy, Sputtered tungsten films on polyimide, an application for X-ray masks, Solid State Electronics 43 (1999) 1075-1078. 
[32] Y.C. Jean, P.E. Mallon, R. Zhang, H. Chen, Y. Li, J. Zhang, Y.C. Wu, T.C. Sandreczki, R. Suzuki, T. Ohdaira, X. Gu, T. Nguyen, Positron studies of polymeric coatings, Rad. Phys. Chem. 68 (2003) 395-402

[33] L. D. Hulett, S. Wallace, J. Xu, B. Nielsen, Cs. Szeles, K.G. Lynn, J. Pfau, A. Schaub, Assay of weathering effects on protective polymer coating using positron annihilation spectroscopy, Appl. Surf. Sci. 85 (1995) 334-338.

[34] C. Degueldre, Ch. Hellwig, Study of a zirconia based inert matrix fuel under irradiation, J. Nucl. Mater. 320 (2003) 96-105

[35] N. Baclet, B. Oudot, R. Grynszpan, L. Jolly, B. Ravat, P. Faure, L. Berlu, G. Jomard, Selfirradiation effects in plutonium alloys, J. All. Comp. (2007) in press.

[36] R.I. Grynszpan, N. Baclet, A. Darque, J.L. Flament, F. Zielinski, W. Anwand, G. Brauer, Positron and deuteron depth profiling in helium-3-implanted electrum-like alloy, Appl. Surf. Sci. 252 (2006) 3252-3255.

[37] R.I. Grynszpan, G. Brauer, W. Anwand, Positron Annihilation in ion-implanted Yttriastabilized zirconia, Mater. Res. Soc. Symp. Proc. 908E (2006) 1-9.

[38] F. Ziegler, J.P. Biersack, The stopping and range of ions in matter, http://www.SRIM.org

[39] R. Krause-Rehberg, F. Börner, F. Redmann, J. Gebauer, R. Kögler, R. Kliemann, W. Skorupa, W. Egger, G. Kögel, W. Triftshäuser, Identification of getter defects in highenergy self-implanted silicon at $\mathrm{Rp}=1 / 2$, Physica B 308 (2001) 442-445.

[40] P.G. Coleman, C.P. Burrows, A.P. Knights, Simple expression for vacancy concentrations at half ion range following $\mathrm{MeV}$ ion implantation of silicon, Appl. Phys. Lett. 80 (2002) 947949.

[41] T. Höchbauer, A. Misra, M. Nastasi, K. Henttinen, T. Suni, I. Suni, S.S. Lau, W. Ensinger, Comparison of thermally and mechanically induced Si layer transfer in hydrogen-implanted $\mathrm{Si}$ wafers, Nucl. Instrum. Meth. B 16 (2004) 257-263.

[42] A.A. Kloidt, H. J. Stock, U. Kleineberg, T. Döhring, M. Pröpper, B. Schmiedeskamp, U. Heinzmann, Smoothing of interfaces in ultrathin $\mathrm{Mo} / \mathrm{Si}$ multilayers by ion bombardment, Thin Solid Films 228 (1993) 154-157.

[43] M. Putero-Vuaroqueaux, H. Fa1k, B. Vidal, A comparative study of the interfacial roughness correlation and propagation in $\mathrm{Mo} / \mathrm{Si}$ multilayers deposited using RF-magnetron sputtering on silicon, ule and zerodur substrates, J. Phys.: Condens. Matter 14 (2002) 8955-8968

[44] H. Faik-Etienne, Etude de l'implantation ionique dans les miroirs multicouches Mo/Si: applications aux optiques diffractives, Thesis INSA-Toulouse (2005).

[45] D. Schödlbauer, G. Kögel, P. Sperr, W. Triftshäuser, Lifetime measurements with a pulsed slow positron beam, Phys. Stat. Sol. (a) 102 (1987) 549-554.

[46] R. Krause-Rehberg, S. Sachert, G. Brauer, A. Rogov, K. Noack, EPOS - An intense positron beam project at the ELBE radiation source in Rossendorf, Appl. Surf. Sci. 252 (2006) 31063110 .

[47] N. Alberola, T. Anthonioz, A. Badertscher, C. Bas, A.S. Belov, P. Crivelli, S.N. Gninenko, N.A. Golubev, M.M. Kirsanov, A. Rubbia, D. Sillou, Development of a high-efficiency slow positron beam for measurements with orthopositronium in vacuum, Nucl. Instrum. Meth. A 560 (2006) 224-232.

[48] K.G. Lynn, W.E. Frieze, P.J. Schultz, Measurement of the positron surface-state lifetime for Al, Phys. Rev. Lett. 52 (1984) 1137-1140.

[49] Depth-Selective Positron Nano-Porosimetry System PALS-1. For further details, see: http://www.fuji-imvac.co.jp/English/PALS.htm. 